\title{
Ethnic variation in personality disorder: evaluation of 6 years of hospital admissions
}

\author{
A. Hossain, ${ }^{1}$ M. Malkov, ${ }^{2}$ T. Lee, ${ }^{3}$ K. Bhui ${ }^{2,4}$
}

BJPsych Bulletin (2018) 42, 157-161, doi:10.1192/bjb.2018.31

${ }^{1}$ North East London National Health Service Foundation Trust; ${ }^{2}$ East London National Health Service Foundation Trust; ${ }^{3}$ Deancross Personality Disorder Service, East London National Health Service Foundation Trust; ${ }^{4}$ Queen Mary University of London

Correspondence to Tennyson Lee (tennyson.lee1@nhs.net)

First received 5 Feb 2018, final revision 5 Apr 2018, accepted 6 Apr 2018

(c) The Authors 2018. This is an Open Access article, distributed under the terms of the Creative Commons Attribution-NonCommercialNoDerivatives licence (http:// creativecommons.org/licenses/by-ncnd/4.0/), which permits non-

commercial re-use, distribution, and reproduction in any medium, provided the original work is unaltered and is properly cited. The written permission of Cambridge University Press must be obtained for commercial re-use or in order to create a derivative work.
Aims and method There is limited evidence on ethnic differences in personality disorder prevalence rates. We compared rates of people with personality disorder admitted to hospital in East London from 2007 to 2013.

Results Of all people admitted to hospital, 9.7\% had a personality disorder diagnosis. The admission rate for personality disorder has increased each year. Compared with White subjects, personality disorder was significantly less prevalent among Black and other minority ethnic (BME) groups. Personality disorder was diagnosed in $20 \%$ of forensic, $11 \%$ of general adult, $8 \%$ of adolescent and $2 \%$ of old-age in-patients.

Clinical implications The increasing number of personality disorder diagnoses year on year indicates the increasing impact of personality disorder on in-patient services. It is important to identify and appropriately manage patients with a personality disorder diagnosis due to the significant strain they place on resources. The reasons for fewer admissions of BME patients may reflect alternative service use, a truly lower prevalence rate or under-detection.

Declaration of interest None.
Personality disorders are characterised by enduring maladaptive patterns of behaviour, cognition and inner experience, exhibited across many domains and deviating markedly from those accepted by the individual's culture. ${ }^{1}$ Comorbidity of personality disorder with other mental disorders is common, and the presence of personality disorder often has a negative effect on treatment outcome. Personality disorder is associated with premature mortality and suicide ${ }^{2}$ and people with the disorder often use services heavily, ${ }^{3}$ leading to calls for improved identification in clinical practice. ${ }^{4}$

\section{Prevalance of personality disorder}

The prevalence of personality disorder increases with levels of care. In the community, estimates range from $4.4 \%$ in the UK, ${ }^{5} 6.1 \%$ in a World Health Organization (WHO) study across 13 countries, ${ }^{6}$ to $8.6 \%$ in Bangalore. ${ }^{7}$ Prevalence of personality disorder is $24 \%$ in the UK at the primary care level. ${ }^{8}$ At the secondary care level, psychiatric out-patient prevalence rates varied between 40 and $92 \%$ in Europe, $45-51 \%$ in the USA and $60 \%$ in Pakistan. ${ }^{9}$

Personality disorder is under-diagnosed in routine practice compared with when structured instruments are used. ${ }^{10}$ A USA study showed $31 \%$ of psychiatric in-patients met criteria for personality disorder, but only $12.8 \%$ of them had a chart diagnosis of personality disorder. ${ }^{11}$ In the UK, there is a reported prevalence rate of $7 \%$ of admissions in general adult psychiatry wards based on routine case note diagnosis. ${ }^{12}$

\section{Review of literature}

We searched the PubMed, PscyInfo and EMBase databases using the search strategy 'personality' AND 'disorder' AND 'prevalence and ethni ${ }^{*}$. We found 10 relevant results and hand-searched references of these papers for additional relevant studies. A meta-analysis (which identified 391 relevant publications and finally included 14) showed significant differences in prevalence between different ethnic groups, raising the question of whether there is a neglect of diagnosis in some ethnic groups or whether these are genuinely differing rates. However, the study does highlight the paucity of research into the prevalence rates of personality disorder among different ethnic minorities. ${ }^{13}$ A study based on a national household survey suggests that the prevalence of personality disorder is at least similar in minority populations to the native population within the UK. ${ }^{14}$

\section{Local context}

London is one of the most ethnically diverse cities in the world, and East London is the most ethnically diverse part of London with $73 \%$ of the population being non-native in 
origin. East London contains 8 out of the top 15 constituencies in the UK with the highest diversity index scores, ${ }^{15}$ making it a useful area for investigating whether there is an ethnic variation in prevalence of illness. Within the data gathering period, East London National Health Service Foundation Trust provided services to three boroughs - Tower Hamlets, Newham and City and Hackney - comprising a population of 815 $000{ }^{16}$ This audit and service evaluation was undertaken in partnership with the Trust as a quality improvement initiative.

\section{Objectives}

The objectives of this audit were:

1. to describe the ethnic variation of psychiatric in-patients with a personality disorder diagnosis in East London;

2. to contrast services such as old age, adolescent, forensic and general adult services.

\section{Method}

Anonymised data from routine service contact were collected from the Trust's electronic patient record system on all admissions between April 2007 and April 2013. Ethnicity categories from the 2001 UK census were used. These data were then compared to census data of local demographics from the census data of 2011. Individual identifiers were not examined because routine clinical data were used in aggregate. As this was a service audit to inform our quality improvement initiatives, ethical approvals were deemed to not be necessary.

\section{Results}

Out of a total of 19102 in-patient admissions in 6 years across three boroughs in all services, 1853 of them had or were eventually given a diagnosis of a personality disorder, which gives us a mean prevalence estimate of $9.7 \%$. Of these in-patients, $56 \%$ were female and $44 \%$ male. This mean prevalence varied from $3 \%$ in Indian and Pakistani populations, to $17 \%$ in the native White British population (Table 1). There is a statistically significant lower prevalence of personality disorder in all ethnicities compared with the White British population, except in those of mixed race heritage where the sample size is too small. There was little variation in personality disorder diagnosis rates between Black and other minority ethnic (BME) groups where there was a sufficiently large sample size.

Table 2 shows the breakdown of the prevalence of personality disorder diagnosis in the different directorates of the Trust. The prevalence was $20 \%$ in forensic, $11 \%$ in general adult, $8 \%$ in adolescent services and $2 \%$ in old-age in-patients. Table 3 compares admission rates to the local population levels of each ethnicity.

The number of people admitted to hospital with a diagnosis of personality disorder has increased year on year, nearly doubling at the end of the 6 year period (Fig. 1).

\section{Discussion}

Our analysis of in-patients in East London demonstrated a 9.7\% prevalence rate of personality disorder, which is in line with previous studies of in-patients in the UK. ${ }^{12}$

\begin{tabular}{|c|c|c|}
\hline Ethnicity & $\begin{array}{l}\text { Prevalence of } \\
\text { personality disorder }\end{array}$ & Odds ratio $(95 \% \mathrm{Cl})$ \\
\hline White British & $17 \%$ & 1 \\
\hline Irish & $7 \%$ & $0.3635(0.25-0.52)$ \\
\hline Black African & $4 \%$ & $0.19(0.105-0.24)$ \\
\hline Black Caribbean & $4 \%$ & $0.22(0.17-0.28)$ \\
\hline Chinese & $4 \%$ & $0.17(0.06-0.56)$ \\
\hline Indian & $3 \%$ & $0.17(0.11-0.25)$ \\
\hline Bangladeshi & $4 \%$ & $0.22(0.17-0.28)$ \\
\hline Pakistani & $3 \%$ & $0.28(0.19-0.419)$ \\
\hline White/Asian mix & $20 \%$ & $1.192(0.68-2.11)$ \\
\hline $\begin{array}{l}\text { White/Black } \\
\text { African mix }\end{array}$ & $1 \%$ & $0.0638(0.009-0.46)$ \\
\hline $\begin{array}{l}\text { White/Black } \\
\text { Caribbean mix }\end{array}$ & $16 \%$ & $0.8904(0.62-1.28)$ \\
\hline Other mix & $14 \%$ & $0.799(0.54-1.18)$ \\
\hline Other Asian & $11 \%$ & $0.6(0.46-0.9)$ \\
\hline Other Black & $7 \%$ & $0.38(0.29-0.49)$ \\
\hline Other White & $8 \%$ & $0.408(0.34-0.88)$ \\
\hline Other ethnicity & $7 \%$ & $0.36(0.28-0.47)$ \\
\hline
\end{tabular}

Although our results indicate little variation in personality disorder rates between different BME groups, they consistently show lower rates compared to the White British population. Lower rates of referrals for BME groups to the local personality disorder service have also been found. ${ }^{17}$ Our findings raise key questions in light of international and national data pointing to the contrary (e.g. the WHO study across 13 countries that found that personality disorder is no less prevalent outside 'westernised' countries ${ }^{6}$ and the UK survey ${ }^{14}$ ). However, the lower incidence of personality disorder presentations in psychiatric emergencies in ethnic minorities has been noted before. ${ }^{18}$

Possible reasons for our findings may include that BME community structures contain the mild to moderate presentations of the disorder, meaning that only those people with extreme cases present to mental health services. BME communities also have difficulties in accessing healthcare, more complex pathways to specialist treatment ${ }^{19}$ and lower rates of accessing healthcare than the majority of the population. $^{20}$

Table 2 Prevalence of personality disorder diagnosis in adult, child and adolescent, old-age and forensic services

\begin{tabular}{lc} 
Directorate & Prevalence of patients \\
\hline Adults & $11 \%$ \\
\hline Child and adolescent & $8 \%$ \\
\hline Forensic & $20 \%$ \\
\hline Old-age service & $2 \%$ \\
\hline
\end{tabular}




\begin{tabular}{|c|c|c|c|c|c|c|c|c|c|c|c|c|c|c|c|c|c|c|}
\hline & 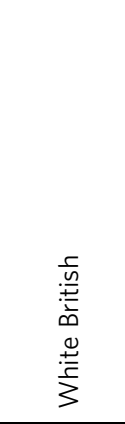 & 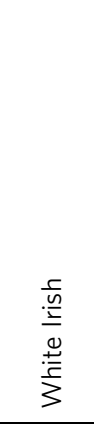 & 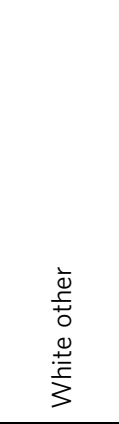 & 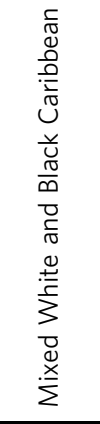 & 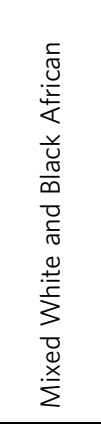 & 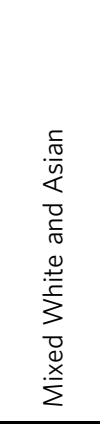 & 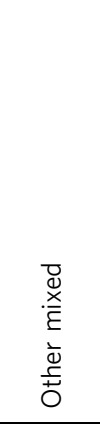 & 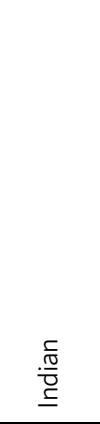 & 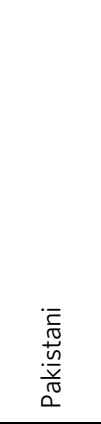 & $\begin{array}{l}\frac{\bar{c}}{\bar{n}} \\
\frac{0}{0} \\
\frac{\pi}{00} \\
\frac{0}{0} \\
\infty\end{array}$ & 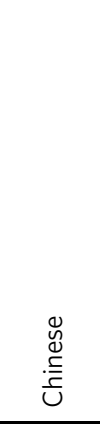 & $\begin{array}{l}\frac{.}{.0} \\
\frac{1}{4} \\
\bar{\Phi} \\
\frac{ \pm}{ \pm} \\
0\end{array}$ & 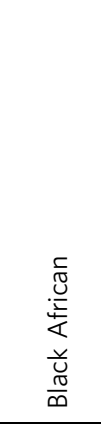 & 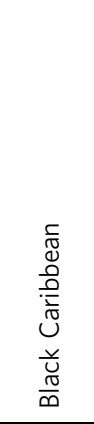 & 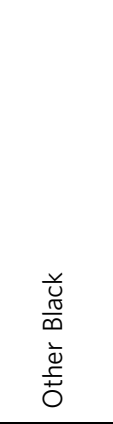 & 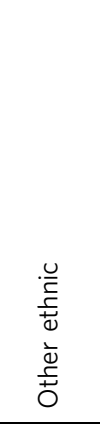 & 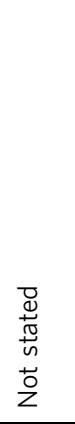 & $\begin{array}{l}\overline{\sqrt{\pi}} \\
\stackrel{0}{0}\end{array}$ \\
\hline \multirow[t]{2}{*}{ General population } & 224029 & 12563 & 107895 & 11830 & 7740 & 8778 & 12151 & 57095 & 34679 & 125060 & 15747 & 32667 & 75389 & 39614 & 20960 & 29690 & & 815734 \\
\hline & $27.46 \%$ & $1.54 \%$ & $13.23 \%$ & $1.45 \%$ & $0.95 \%$ & $1.08 \%$ & $1.49 \%$ & $7.00 \%$ & $4.25 \%$ & $15.33 \%$ & $1.93 \%$ & $4.00 \%$ & $9.24 \%$ & $4.86 \%$ & $2.57 \%$ & $3.64 \%$ & & \\
\hline Number of in-patients & 6374 & 473 & 1921 & 232 & 77 & 76 & 212 & 696 & 490 & 1611 & 84 & 569 & 2311 & 1660 & 885 & 851 & 580 & 19102 \\
\hline Percentage of in-patients & $33 \%$ & $2 \%$ & $10 \%$ & $1 \%$ & $0 \%$ & $0 \%$ & $1 \%$ & $4 \%$ & $3 \%$ & $8 \%$ & $0 \%$ & $3 \%$ & $12 \%$ & $9 \%$ & $5 \%$ & $4 \%$ & $3 \%$ & \\
\hline Number of patients with personality disorder & 1090 & 33 & 149 & 36 & 1 & 15 & 30 & 23 & 17 & 69 & 3 & 63 & 89 & 73 & 64 & 59 & 39 & 1853 \\
\hline
\end{tabular}


Number of yearly admissions

with personality disorder

$07 / 08-192(6.5 \%)$

$08 / 09-251(8.8 \%)$

$09 / 10-295(9.3 \%)$

$10 / 11-322(10 \%)$

$11 / 12-396(11 \%)$

$12 / 13-397(11 \%)$

Rates of non-native personality disorder admissions

$07 / 08 \quad 22 \%$

$08 / 09 \quad 18 \%$

$09 / 10 \quad 19 \%$

$10 / 11 \quad 26 \%$

$11 / 12 \quad 27 \%$

$12 / 13 \quad 30 \%$

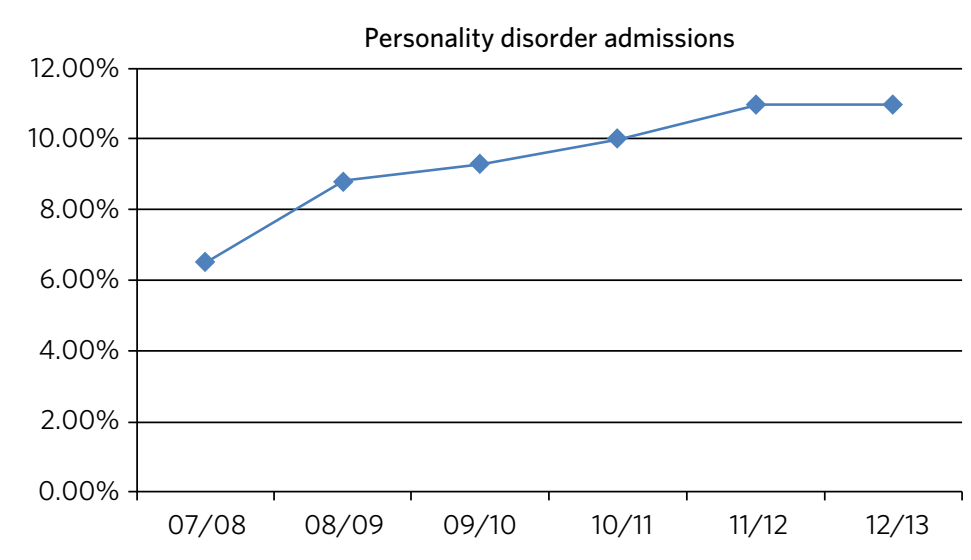

Fig. 1 Number of yearly admissions of people with personality disorder.

There is some evidence that there are ethnic variations in the presentation of the disorder, ${ }^{21,22}$ that specific symptoms can be shaped by culture ${ }^{23}$ and that individuals of differing ethnicity may present with different patterns of personality disorder pathology. ${ }^{24,25}$ In the key population group in East London, there is insufficient consistent evaluation into prevalence, recognition and service access for people with personality disorder from Asian populations ${ }^{26}$ and studies showing low rates of personality disorder in Asian-origin samples may be a result of a lack of understanding of what constitutes personality and personality disorder in Asian culture. ${ }^{27}$ Differences in the presentation of symptoms of personality disorder in different cultures would not adequately be screened for by the tools currently in use. The preceding factors raise the possibility of misdiagnosis and suboptimal treatment. ${ }^{28}$ In addition, 'reverse racism' may be occurring, with psychiatrists reluctant to make a diagnosis of personality disorder because it may be perceived as racist.

The annually increasing number of personality disorder diagnoses may reflect an increased willingness to diagnose this condition due to the increase in evidence-based treatment and the publication of National Institute for Health and Care Excellence guidelines on personality disorders in 2009. However, it is interesting to note that the proportion of patients admitted under sections of the Mental Health Act (2007) (MHA) has been steadily increasing since at least 2009 (http://www.cqc.org.uk/publications/majorreport/monitoring-mental-health-act-report\#old-reports), and there could possibly be a correlate, especially after the changes introduced to the act in 2007. ${ }^{29}$ Our analysis did not pick out whether the people diagnosed with personality disorder were informal or under a section of the MHA.

The prevalence of $8 \%$ of adolescent in-patients with a diagnosis of a personality disorder is remarkable, as ICD-10 (1992) discourages the diagnosis in under $18 \mathrm{~s}^{30}$ This suggests that clinicians may find the diagnosis of heuristic value. There has been considerable evidence that the diagnostic criteria for borderline personality disorder (and other personality disorders) are as valid, reliable and stable before age 18 as after age $18 .^{31}$

The prevalence of personality disorder among older people in the community has been estimated to be about
10\%. ${ }^{32}$ Among older in-patients, personality disorder has been seen in $6 \%$ of those with organic mental disorders and $24 \%$ of those with major depressive disorder. ${ }^{33}$ Our finding of a $2 \%$ prevalence suggests that personality disorder may be under-diagnosed significantly in routine practice in old-age patients.

\section{Limitations}

Data were collected from one Trust in the UK. However, it is the most ethnically diverse one (Census 2011) ${ }^{16}$, and there is no reason to expect differences in routine diagnostic practice in other Mental Health Trusts in the UK. We do not anticipate problems relating to quality and validity of the personality disorder data compared with other diagnostic groups because all diagnoses are made on the basis of routine clinical care provided by the Trust.

\section{Recommendations}

The significant and rising proportion of in-patients diagnosed with personality disorder, combined with cost and pressures on in-patient beds, indicates that variations in recognition, access and management of these patients needs to be understood to ensure accurate identification and an improvement in present services.

Research targeting reasons for the lower diagnostic rates of personality disorder in BME groups could include whether there are cultural norms shared between BME communities that limit seeking help from mental health services for symptoms of personality disorder, whether there are variations in pathways to care, or whether there are variations in the attitudes of clinicians in diagnosing personality disorder in different ethnic groups.

The high proportion of adolescent in-patients diagnosed with personality disorder highlights the importance of a good transition from Child and Adolescent Mental Health Services to adult services, especially given difficulties these patients have with attachment. The ongoing presence of personality disorder in old-age services indicates the need for expertise in detecting and managing this diagnosis in these services, as these patients may represent the most difficult 
of personality disorder presentations in terms of not having 'burnt out' as is often expected.

There is a role for well-designed databases that lend themselves to ongoing analyses of routinely collected clinical data reflecting real service activity. All our results and inferences were obtained from such data, which provides us a lowcost opportunity for comparison over time and in different regions. ${ }^{34}$ These data inform our quality improvement actions to improve clinical skills in assessment and management of personality disorder, and to better understand the needs of adolescents and elderly people with personality disorder.

\section{Acknowledgements}

We thank the staff at the Information Department of the East London National Health Service Foundation Trust for their excellent help in routine data retrieval. A poster presentation of preliminary results was presented at the 3rd World Congress of Cultural Psychiatry in London on 9-11 Mar 2012.

\section{About the authors}

A. Hossain, MRCPsych, Consultant Psychiatrist, North East London National Health Service Foundation Trust, UK; M. Malkov, MRCPsych, ST5 CAMHS Specialty Spr, Tavistock and Portman NHS Foundation Trust, UK; T. Lee FFCH, MRCPsych, Consultant Psychiatrist in Psychotherapy, Deancross Personality Disorder Service, East London National Health Service Foundation Trust, UK; K. Bhui, MD, FRCPsych, Professor of Cultural Psychiatry and Epidemiology, Queen Mary University of London and Honorary Consultant Psychiatrist, East London National Health Service Foundation Trust, UK.

\section{References}

1 Potter N. Mapping the Edges and the In-Between: A Critical Analysis of Borderline Personality Disorder. International Perspectives in Philosophy and Psychiatry. Oxford University Press, 2013.

2 Tyrer P, Reed GM, Crawford MJ. Classification, assessment, prevalence, and effect of personality disorder. Lancet 2015; 385: 717-26.

3 Bender DS, Dolan RT, Skodol AE, Sanislow CA, Dyck IR, McGlashan TH, et al. Treatment utilization by patients with personality disorders. Am J Psychiatry 2001; 158: 295-302.

4 Soetman DI, Hakkaart-van Roijen L, Verheul R, Busschbach JJ. The economic burden of personality disorders in mental health care. J Clin Psychiatry 2008; 69: 259-65.

5 Coid J, Yang M, Tyrer P, Roberts A, Ullrich S. Prevalence and correlates of personality disorder in Great Britain. Br J Psychiatry 2006; 188: 423-31.

6 Huang Y, Kotov R, de Girolamo G, Preti A, Angermeyer M, Benjet C, et al. DSM-IV personality disorders in the WHO World Mental Health Surveys. Br J Psychiatry 2009; 195: 46-53.

7 Sharan P. An overview of Indian research in personality disorders. Indian J Psychiatry 2010; 52: 250-4.

8 Moran P, Jenkins R, Tylee A, Blizard R, Mann A. The prevalence of personality disorder among UK primary care attenders. Acta Psychiatr Scand 2000; 102: 55-7.

9 Beckwith H, Moran PF, Reilly J. Personality disorder prevalence in psychiatric outpatients: a systematic literature review. Personal Ment Health 2014; 8: 91-101.

10 Zimmerman M, Mattia Jl. Differences between clinical and research practices in diagnosing borderline personality disorder. Am J Psychiatry 1999; 156: 1570-4.

11 Leontieva L, Gregory R. Characteristics of patients with borderline personality disorder in a state psychiatric hospital. J Pers Disord 2013; 27: 222-32.
12 Dasgupta P, Barber J. Admission patterns of patients with personality disorder. Psychiatr Bull 2004; 28: 321-32.

13 McGilloway A, Hall RE, Lee T, Bhui KS. A systematic review of personality disorder, race and ethnicity: prevalence, aetiology and treatment. BMC Psychiatry 2010; 10: 33.

14 Crawford M, Rushwaya T, Bajaj P, Tyrer P, Yang M. The prevalence of personality disorder among ethnic minorities: findings from a national household survey. Personal Ment Health 2012; 6: 175-82.

15 Census Information Scheme. 2011 Census Snapshot: Ethnic Diversity Indices. Update CIS2012-04. Census Information Scheme, GLA Intelligence, 2012. Available at: http://data.london.gov.uk/dataset/2011census-diversity/resource/8a7e717c-4fe7-446a-bb79-d425e105ee97.

16 Office for National Statistics. Office for National Statistics (ONS) Census, 2011. Available at: https://www.nomisweb.co.uk/census/2011/ks201ew.

17 Garrett C, Lee T, Blackburn S, Priestly L, Bhui K. Personality Disorder: challenges in service development in the light of the new NICE guidelines for Personality Disorder. Psychiatrist 2011; 35: 22-6.

18 Tyrer $\mathrm{P}$, Merson S, Onyett $\mathrm{S}$, Johnson T. The effect of personality dis order on clinical outcome, social networks and adjustment: a controlled clinical trial of psychiatric emergencies. Psychol Med 1994; 24: 731-40.

19 Bhui K, Stansfeld S, Hull S, Priebe S, Mole F, Feder G. Ethnic variations in pathways to and use of specialist mental health services in the UK Systematic review. Br J Psychiatry 2003; 182: 105-16.

20 Sashidharan SP. Inside Outside: Improving Mental Health Services for Black and Minority Ethnic Communities in England. Department of Health National Institute for Mental Health in England, 2003.

21 Selby EA, Joiner T. Ethnic variations in the structure of borderline personality disorder symptomatology. J Psychiatr Res 2008; 43: 115-23.

22 Manseau M, Case BG. Racial-ethnic disparities in outpatient mental health visits to U.S. physicians, 1993-2008. Psychiatr Serv 2014; 65: 59-67.

23 Paris J, Lis E. Can sociocultural and historical mechanisms influence the development of borderline personality disorder? Transcult Psychiatry 2013; 50: 140-51.

24 Iwamasa G, Larrabee AL, Merritt RD. Are personality disorder criteria ethnically biased? A card sort analysis. Cultur Divers Ethnic Minor Psychol 2000; 6: 284-96.

25 Chavira DA, Grilo CM, Shea MT, Yen S, Gunderson JG, Morey LC, et al. Ethnicity and four personality disorders. Compr Psychiatry 2003; 44: 483-91.

26 de Bernier GL, Kim YR, Sen P. A systematic review of the global prevalence of personality disorders in adult Asian populations. Personal Ment Health 2014; 8: 264-75.

27 Ryder AG, Sun J, Dere J, Fung K. Personality disorders in Asians: summary, and a call for cultural research. Asian J Psychiatr 2014; 7: 86-8.

28 De Genna NM, Feske U. Phenomenology of borderline personality disorder: the role of race and socioeconomic status. J Nerv Ment Dis 2013; 201: 1027-34.

29 Mental Health Act 2007, chapter 12.

30 World Health Organization. The ICD-10 Classification of Mental and Behavioural Disorders: Clinical Descriptions and Diagnostic Guidelines World Health Organization, 1992.

31 Chanen AM, McCutcheon LK, Jovey M, Jackson HJ, McGorry PD. Prevention and early intervention for borderline personality disorder. Med J Aust 2007; 187: S18-21.

32 Abrams RC, Horowitz SV. Personality disorders after age 50: a meta-analysis. J Personal Disord 1996; 10: 271-81.

33 Kunik ME, Mulsant BH, Rifai AH, Sweet RA, Pasternak R, Zubenko GS. Diagnostic rate of comorbid personality disorder in elderly psychiatric inpatients. Am J Psychiatry 1994; 151: 603-5.

34 Kane R, Wellings K, Free C, Goodrich J. Uses of routine data sets in the evaluation of health promotion interventions: opportunities and limitations. Health Educ 2000; 100: 33-41.

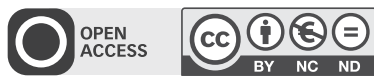

